\title{
SINTERING SHAPE DISTORTIONS CONTROLLED BY INTERFACE ROUGHNESS IN POWDER COMPOSITES
}

\author{
D. Giuntini ${ }^{\text {a }}{ }^{*}$, I.-W. Chen ${ }^{\text {b }}$, E.A. Olevsky ${ }^{\text {a }}$ \\ ${ }^{a}$ Department of Mechanical Engineering, San Diego State University, \\ 5500 Campanile Drive, San Diego, CA 92182, USA \\ ${ }^{\mathrm{b}}$ Department of Materials Science and Engineering, University of Pennsylvania, \\ 3231 Walnut Street, Philadelphia, PA 19104, USA
}

The co-sintering of laminar composites is modeled by means of finite element simulations. The densification of an alumina/ceria-stabilized tetragonal zirconia polycrystal bilayer is optimized by substituting the conventional flat interface morphology with a wavy contact surface. The presence of such roughness significantly decreases the undesired macroscopic distortions characterizing multi-material sintering. Various configurations of the wavy interface are numerically reconstructed. The introduction of rough interfaces generates beneficial stress redistribution and constraint relaxation effects, thus reducing the bending of the composite. The obtained results are in good agreement with previously conducted experiments and provide new guidelines to prevent flaw generation.

Keywords: Sintering; Multilayers; Finite Element Analysis; Shape Instabilities.

The production of laminar composites is of high interest for a variety of applications, from structural components to solid oxide fuel cells, and has therefore been addressed with several different approaches. A common strategy involves sintering technologies coupled with suitable pre-consolidation processes [14]. Even though widely experimented, this kind of procedure still needs to be optimized, since shape distortions and instability issues oftentimes arise. The simultaneous sintering of materials with distinct shrinkage rates is the source of non-negligible deformations and consequent internal stresses, crack nucleation and propagation, residual porosity and segregation phenomena, with a significant degradation of the final composite properties [5-12].

Several studies have been dedicated to co-sintering processes, involving experimental campaigns, analytical modeling and numerical simulations. Comprehensive investigations, typically considering a

\footnotetext{
* Corresponding author. Email address: dgiuntini@mail.sdsu.edu
} 
shrinking layer fixed to a fully dense substrate, have revealed the fundamentals of flaw formation mechanisms in constrained sintering [13-21]. More specific models on the consolidation of bilayered systems have subsequently been proposed, in which the stress distributions and interface conditions have been correlated with the densification anisotropy [22-28]. Together with the inhomogeneities due to the combination of different materials, the influence of gravity and green density gradients have also been analyzed [29-32]. The most recent advancements consist in the reconstruction of bilayer or multilayer composites sintering with numerical tools, namely kinetic Monte Carlo, discrete and finite element modeling [33-38].

Alumina and zirconia are among the materials that are most frequently employed for the synthesis of laminar composites [39-43]. It is in this context that Chen et al. have developed some skillful strategies in order to individuate and exploit the beneficial effects of these unavoidable interface instabilities characterizing multilayered materials [44-47]. They found that by allowing the interfaces among the different layers to attain a corrugated morphology, by means of repeated folding and rolling of the bimaterial medium, the subsequent sintering step was greatly improved, showing no cracks and overall improved mechanical properties. More recent works have further confirmed the efficacy of this approach $[48,49]$.

The present study is devoted to a more detailed and quantitative analysis of such a phenomenon. Only a limited control over the formation of interfacial waviness is possible during experimentation, and specific data on this roughness evolution and influence on densification are not accessible. We have, therefore, built a modeling framework in which the sintering of a bimodal composite can be reconstructed.

Numerical simulations are a valuable resource to isolate the various effects that play a role in co-sintering and cannot be studied separately during the actual procedures. Different simplified schematics can be implemented; and experimentally unobtainable quantitative data, on the conditions experienced by the specimen for the whole duration of the sintering process, can be computed.

We employed commercial finite-element software, COMSOL Multiphysics ${ }^{\mathrm{TM}}$, equipped with the respective sintering constitutive equations [50], to reproduce the isothermal sintering of a bilayer 
composite, with characteristics analogous to the laminates considered by Chen et al. in their experiments [44-47]. Namely, $\mathrm{Al}_{2} \mathrm{O}_{3} / \mathrm{Ce}-\mathrm{TZP}\left(\mathrm{Ce}-\mathrm{O}_{2}\right.$-stabilized tetragonal zirconia polycrystal) systems with corrugated interfaces were modeled. The case of flat interface is compared to a square wave-shaped contact surface, varying its dimensions and its extension with respect to the entire bilayer thickness. We assessed the connection between interface roughness features and macroscopic bending of the composite. The positive effects of the interface waviness were confirmed by a reduction in the overall curvature. Once these improvements were assessed, we also explored some additional interface morphologies.

The geometries that we considered are represented in Figure 1. Because of the domain's symmetries, and to lighten the computations, we reconstructed one quarter of a specimen, with dimensions analogous to the experimental cases that we used as references. The dimensions given in the figure are relative to this portion of the composite.

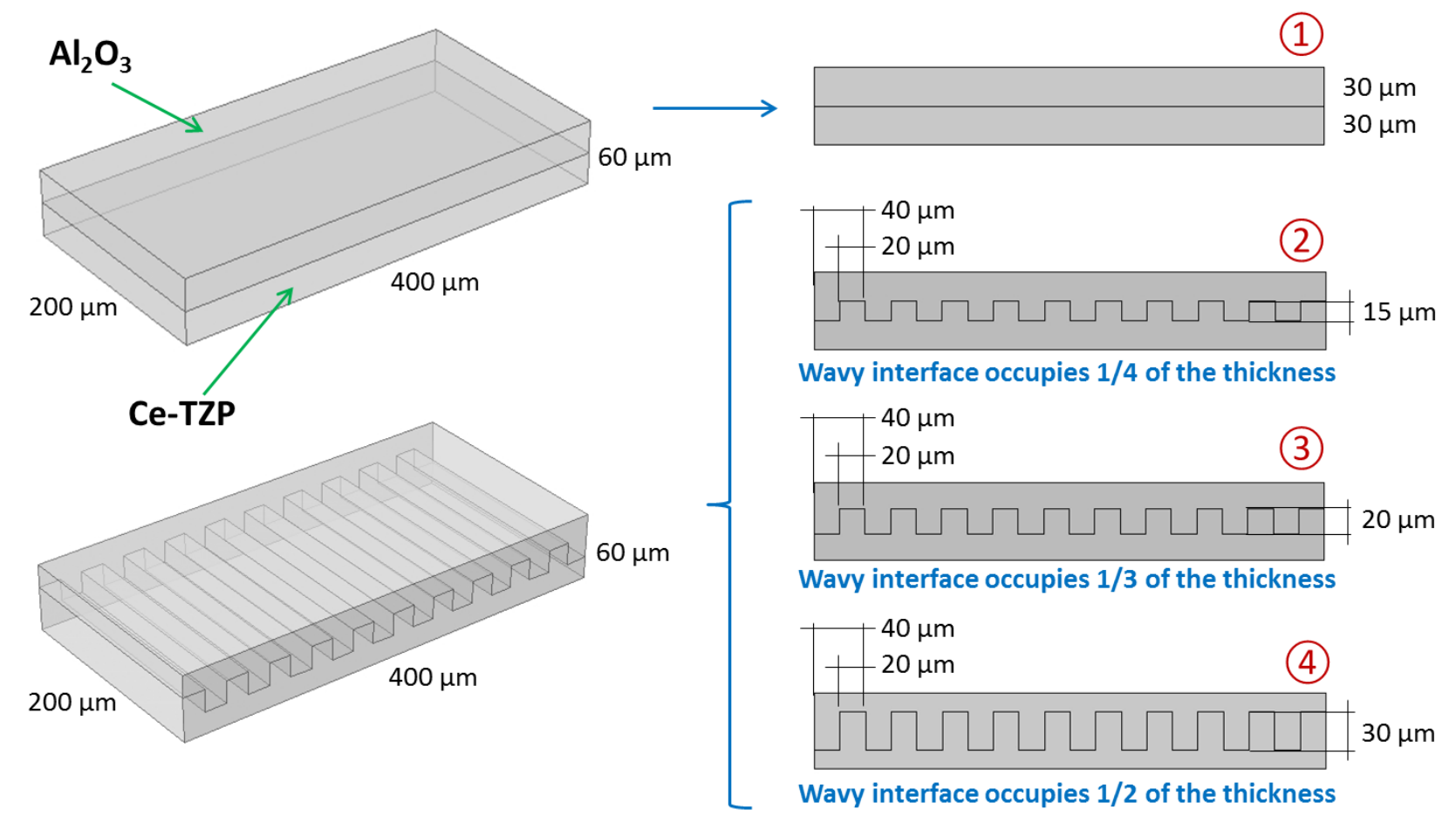

Figure 1. Geometries of the study domains, for the conventional flat interface (1) and the three alternative wavy interface cases $(2,3,4)$. 
As shown in Figure 1, the conventional flat interface geometry, consisting of a specimen evenly split into two layers, was replaced by a square wave-shaped contact surface in three different versions, varying the ratio between the roughness extension in the thickness direction and the thickness value itself, but keeping constant the dimensional parameters in the length direction. The interfacial waviness was directed along the longest side, in which deformations are most notable, in order to obtain the clearest possible data on the bending alterations due to the roughness features.

The top layer was alumina, while the ceria-stabilized zirconia was at the bottom. The shrinkage rates differed significantly, due to the different properties of these two materials.

To simulate the densification and consequent deformation of the bilayer, the continuum theory of sintering was employed [50]. This model requires that the constitutive behavior characterizing the powder particles is specified. For our case of free sintering of micro-sized powders, a linear viscous behavior is the most appropriate choice. The sintering constitutive equation therefore becomes:

$$
\sigma_{i j}=2 \eta_{0}\left[\varphi \dot{\varepsilon}_{i j}+\left(\psi-\frac{\varphi}{3}\right) \dot{e} \delta_{i j}\right]+P_{L} \delta_{i j}
$$

in which $\sigma_{i j}$ is the stress tensor $(\mathrm{Pa}), \dot{\varepsilon}_{i j}$ the strain rate tensor $(1 / \mathrm{s}), \dot{e}$ the first strain rate tensor invariant $(1 / \mathrm{s}), \eta_{0}$ the viscosity of the fully dense material constituting the powder $(\mathrm{Pa} \cdot \mathrm{s}), \varphi$ the normalized shear modulus (1), $\psi$ the normalized bulk modulus (1), $P_{L}$ the sintering stress (Pa), and $\delta_{i j}$ the Kronecker delta. The volume shrinkage rate, $\dot{e}$, is connected to the pores volume fraction, $\theta$, by means of the following continuity equation

$$
\dot{e}=\frac{\dot{\theta}}{(1-\theta)}
$$

The porous material's properties are defined according to the Skorokhod-Olevsky model, as $\varphi=$ $(1-\theta)^{2}, \psi=2(1-\theta)^{3} /(3 \theta)$ and $P_{L}=3 \alpha(1-\theta)^{2} / r_{p}$

In the latter expression, sintering stress is given as directly proportional to the material's surface tension, $\alpha\left(\mathrm{J} / \mathrm{m}^{2}\right)$, since this is the driving force of sintering, and inversely proportional to the powder particles radius, $r_{p}(\mathrm{~m})$. From the equations above it is also evident that the densification rate of a certain material 
is directly proportional to the sintering stress and inversely proportional to its viscosity. In this context, the magnitude of the viscosity of a fully dense material can be determined to be equal to $E / 3$, where $E$ is the Young's modulus $(\mathrm{Pa})$, as proposed by Scherer according to the analogy of the linear viscous and elastic constitutive behaviors [51]. Such analogy states that the solution of a stress problem for a linear viscous material can be immediately inferred from the corresponding elastic problem, by replacing strains with strain rates, and $E$ with $3 \eta_{0}$ (the units of the proportionality constant between elastic modulus and viscosity are thus seconds). Note that, at the sintering temperature used $\left(1600{ }^{\circ} \mathrm{C}\right)$, alumina has both a higher viscosity (104 GPa.s) and a lower surface energy $\left(0.9 \mathrm{~J} / \mathrm{m}^{2}\right)$ than zirconia $\left(43 \mathrm{GPa} \cdot \mathrm{s}\right.$ and $1.5 \mathrm{~J} / \mathrm{m}^{2}$, respectively) [52-54]. This leads to lower shrinkage rates, even though the powder particles sizes were $0.1 \mu \mathrm{m}$ for alumina and $0.2 \mu \mathrm{m}$ for zirconia. The selections of particle size and sintering temperature were based on the work by Chen et al. that inspired the present study. Experiments had also shown that zirconia was the faster-sintering component, as our simulations were able to reproduce [45]. Another fact that was experimentally observed [44-47] and re-encountered in our models was the reduced amount of macroscopic distortions arising with the formation and growth of the interfacial waviness, an effect imputable to the decrease of long-range stresses and to the relaxation of the in-plane constraints responsible for flaw generation and crack propagation.

The isothermal sintering time was chosen to be corresponding to the minimum time necessary to attain full densification of the faster-shrinking layer, without proceeding further. After this point, the shrinkage of the still porous top layer would tend to drag the lower dense layer back towards the initial straight configuration. This stage has a high potential for the nucleation of a variety of defects and instability phenomena, the treatment of which goes beyond the purpose of the present work. The required sintering time resulted to be 2 hours, which is in reasonable agreement with experiments, conducted for 4 hours with multilayers and until full densification of both materials was obtained.

The boundary and initial conditions to complete the process reconstruction consisted in the abovementioned symmetry conditions at the two vertical sides necessary to consider one quarter of the overall 
bilayer composite, fixing a point at the intersection of these same two faces in order to prevent translations of the studied domain, and an initial porosity fraction of $36 \%$ in both layers.

The results of the FEM simulations for the various implemented specimen's geometries are presented in Figure 2. Together with the four geometries shown in Figure 1, we added a case in which again one half of the total sample's thickness is occupied by the wavy interface, but the frequency of the wave is doubled (number 5), and a case of triangular-shaped wave, still with the same amplitude (number 6). The normalized global displacement fields are mapped in the entirety of the analyzed domain and the overall deformation of the bilayer after two hours of sintering is depicted with a scale factor equal to 1 . The normalized displacement is calculated as $\sqrt{u^{2}+v^{2}+w^{2}}$ divided by the initial laminate thickness (60 $\mu \mathrm{m})$, where $u, v$ and $w$ are the displacement components in the length, width and thickness direction, respectively. The same color scale is utilized for all the legends, underlining the differences among the various cases.
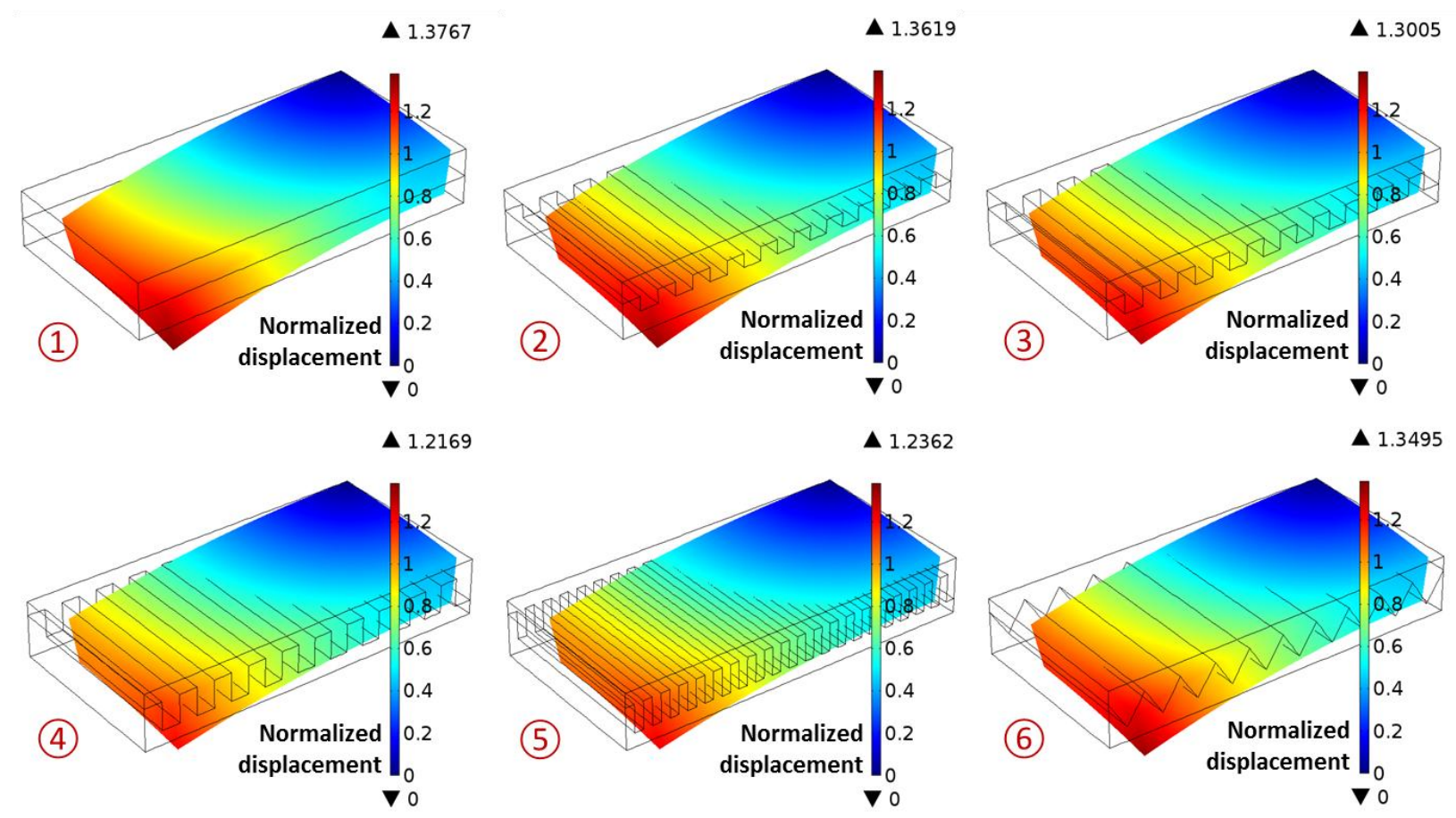

Figure 2. Displacement fields for $\mathrm{Al}_{2} \mathrm{O}_{3} / \mathrm{Ce}$-TZP bilayers with varying interface roughness geometries:

(1) flat interface; (2) 1/4 thickness occupied by roughness; (3) 1/3 thickness occupied by roughness;

(4) $1 / 2$ thickness occupied by roughness; (5) $1 / 2$ thickness occupied by roughness with doubled wave frequency;

(6) $1 / 2$ thickness occupied by triangular wave-shaped interface. 
The beneficial effects of the presence of a wavy interface are evident. By comparing cases 1-4, we see that the curvature of the laminate decreases with increasing extension of the waves in the thickness direction. Raising the number of waves in the length direction while keeping this extension constant, instead, does not provide any positive influence towards bending reduction, and even less advantages are brought when shifting to a triangular morphology. Configurations 5 and 6 will thus be neglected in the continuation of this report.

We calculated estimations of the curvature in the length direction for the various geometries, and plotted them with respect to the ratio between the roughness amplitude and the overall bilayer thickness. Figure 3 provides the respective graphic. Note that the geometrical parameters used to normalize the data are the initial values of bilayer thickness $(\mathrm{H})$ and waviness extension $(\mathrm{t})$, while the radius of curvature $(\mathrm{R})$ is relative to the final shape. From the plot we infer that, by switching from a flat interface to a wavy configuration with a substantial extension (up to 1/2) in the direction perpendicular to the interface midplane, we obtain a curvature reduction of about $20 \%$. The point corresponding to $t / H=1$ is the limiting case of roughness occupying the entire thickness of the laminate, and thus contradicts the definition of laminate itself. This case was simulated purely for comparison, and it is indeed interesting to see that it shows no bending. This further confirms the hypothesis that sintering distortions are caused by the constraint imposed by the interface parallel to the composite layers.

It is important to point out that while the curvature is being significantly impacted by the presence of a rough interface among the two different materials, the porosity values and distributions are instead substantially independent of this feature. In every configuration considered, the top alumina layer had an average $10 \%$ of residual porosity, while the bottom zirconia component had $1 \%$. It is also interesting to note that in a thin area adjacent to the interface, an intermediate value of porosity (about 5\%) was found in all the cases that we investigated. This phenomenon is an additional factor of agreement with the previously conducted experiments and relative post-processing analyses, according to which the sintering stress in the surrounding zone assumes an intermediate value between the single phases' ones. 


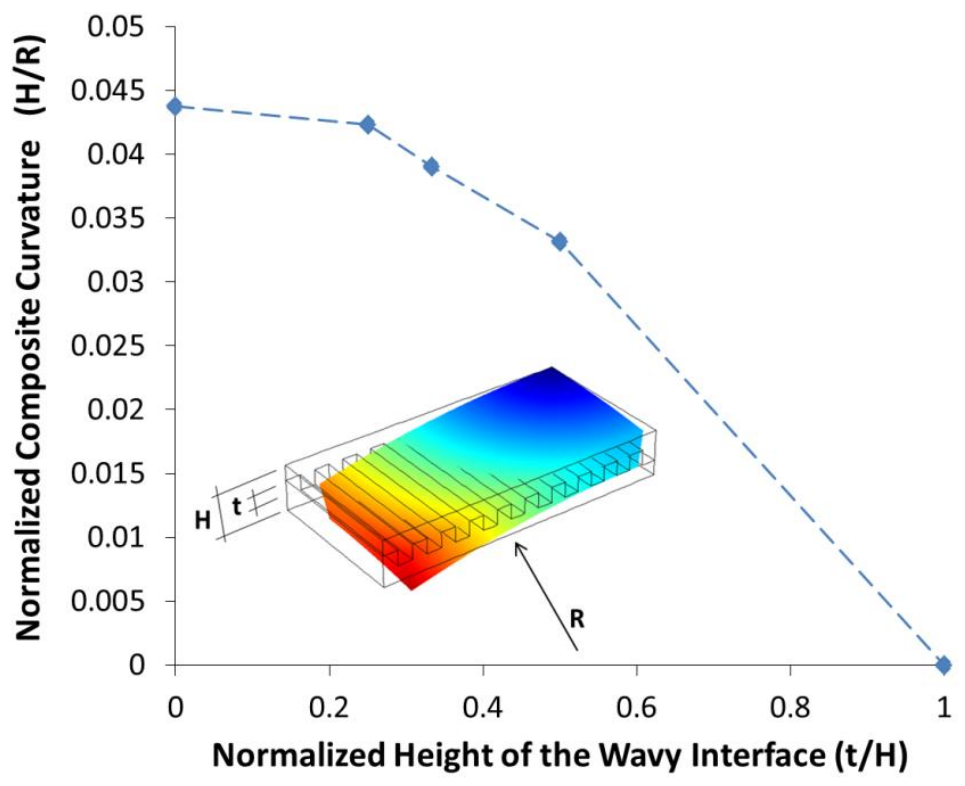

Figure 3. Curvature of the bilayer in function of the roughness extension in the direction of the composite thickness.

Following the hypotheses proposed when this phenomenon was first investigated [44-47], we attribute the change in deformation intensity to a favorable stress redistribution caused by the presence of the wavy interface. A flat interface constitutes a severe constraint in presence of differential shrinkage rates, while a relaxation is provided when portions of the contact area between the two materials are oriented perpendicular to each other at regular intervals. One of the causes of macroscopic bending in multilayered composites is the creation of stress gradients within the cross-sections perpendicular to the interface, which typically range from the tensile stresses of the faster-sintering phase to compressive states in the other component. In the conventional flat interface situation, these conditions propagate throughout the entire length of the laminate material, and thus long-range non-symmetrical stresses are formed, leading to the bending of the composite. The presence of waviness leads to a stress distribution with an alternating sign (with respect to the mid-interface plane), thus relaxing the interface constraint itself and lowering the curvature of the bilayer. The assumption that increasing the roughness of free surfaces of sintered specimens could lead to the possible reduction of sintering shape distortions is a hypothesis to be verified in future investigations. 
The presented results confirm the previously individuated trend, according to which producing laminar composites with wavy interfaces among the different materials (or, alternatively, among layers of the same material with different porosity amounts) leads to a significant decrease in the undesired bending phenomena accompanying co-sintering processes. Indicating that our simulations are in agreement with experimental evidence, the FEM framework developed according to the continuum theory of sintering and already utilized for the solution of other sintering-related issues [55-57], proves its efficacy in this context, as well. The simplified bilayer model allows the separate analysis of the effects of the many parameters involved and the obtainment of quantitative results, both providing additional insights on the process with respect to experiments and useful guidelines for the synthesis of multi-material composites. Room is left for further refinements, investigations and optimizations, and the versatility of this modeling technique renders it suitable for various sintering application.

The support of the National Science Foundation Division of Civil and Mechanical Systems and Manufacturing Innovations (NSF Grant no. CMMI DMREF 1234114) is gratefully appreciated. IWC was supported by the grant DE-SC0007064 funded by the U.S. Department of Energy, Office of Science.

\section{References}

[1] T. Chartier, D. Merle, J.L. Besson, J. Eur. Ceram. Soc. 15 (1995) 101-107.

[2] Q.L. Liu, K.A. Khor, S.H. Chan, X.J. Chen, J. Power Sources 162 (2006) 1036-1042.

[3] G.-B. Jung, C.-J. Wei, A. Su, F.-B. Weng, Y.-C. Hsu, S.-H. Chan, J. Solid State Electrochem. 12 (2008) 1605-1610.

[4] E.A. Olevsky, X. Wang, A. Maximenko, M.A. Meyers, J. Am. Ceram. Soc. 90 (2007) 3047-3056.

[5] T. Cheng, R. Raj, J. Am. Ceram. Soc. 72 (1989) 1649-1655.

[6] W.J. Clegg, Acta Metall. Mater. 40 (1992) 3085-3093. 
[7] O. Sbaizero, E. Lucchini, J. Eur. Ceram. Soc. 16 (1996) 813-818.

[8] A. Atkinson, A. Selçuk, Acta Mater. 47 (1999) 867-874.

[9] M.-J. Chiang, J.-H. Jean, S.-C. Lin, J. Am. Ceram. Soc. 94 (2011) 683-686.

[10] J.-B. Ollagnier, O. Guillon, J. Rödel, J. Am. Ceram. Soc. 93 (2010) 74-81.

[11] J.-B. Ollagnier, D.J. Green, O. Guillon, J. Rödel, J. Am. Ceram. Soc. 92 (2009) 2900-2906.

[12] B. Charlas, H.L. Frandsen, K. Brodersen, P.V. Henriksen, M. Chen, J. Power Sources 288 (2015) $243-252$.

[13] R. k. Bordia, R. Raj, J. Am. Ceram. Soc. 68 (1985) 287-292.

[14] R.K. Bordia, A. Jagota, J. Am. Ceram. Soc. 76 (1993) 2475-2485.

[15] R.K. Bordia, G.W. Scherer, Acta Metall. 36 (1988) 2393-2397.

[16] R.K. Bordia, G.W. Scherer, Acta Metall. 36 (1988) 2399-2409.

[17] D.J. Green, O. Guillon, J. Rödel, J. Eur. Ceram. Soc. 28 (2008) 1451-1466.

[18] Y.-C. Lin, J.-H. Jean, J. Am. Ceram. Soc. 85 (2002) 150-154.

[19] I. Barsoum, K.S. Ravi Chandran, Eng. Fract. Mech. 70 (2003) 2015-2031.

[20] E. Olevsky, A. Molinari, Int. J. Plast. 16 (2000) 1-37.

[21] A. Atkinson, J.-S. Kim, R. Rudkin, S. Taub, X. Wang, J. Am. Ceram. Soc. 94 (2011) 717-724.

[22] P.Z. Cai, D.J. Green, G.L. Messing, J. Am. Ceram. Soc. 80 (1997) 1929-1939.

[23] P.Z. Cai, D.J. Green, G.L. Messing, J. Am. Ceram. Soc. 80 (1997) 1940-1948.

[24] J. Kanters, U. Eisele, J. Rödel, J. Am. Ceram. Soc. 84 (2001) 2757-2763.

[25] H.L. Frandsen, E. Olevsky, T.T. Molla, V. Esposito, R. Bjørk, N. Pryds, J. Am. Ceram. Soc. 96 (2013) 80-89.

[26] T.T. Molla, D.K. Ramachandran, D.W. Ni, V. Esposito, F. Teocoli, E. Olevsky, R. Bjørk, N. Pryds, A. Kaiser, H.L. Frandsen, J. Eur. Ceram. Soc. 35 (2015) 941-950.

[27] D.W. Ni, E. Olevsky, V. Esposito, T.T. Molla, S.P.V. Foghmoes, R. Bjørk, H.L. Frandsen, E. Aleksandrova, N. Pryds, J. Am. Ceram. Soc. 96 (2013) 2666-2673. 
[28] T.T. Molla, H.L. Frandsen, R. Bjørk, D.W. Ni, E. Olevsky, N. Pryds, J. Eur. Ceram. Soc. 33 (2013) 1297-1305.

[29] J. Lu, H.H. Hng, X. Song, T. Zhang, J. Ma, J. Am. Ceram. Soc. 94 (2011) 1528-1535.

[30] S.E. Schoenberg, D.J. Green, A.E. Segall, G.L. Messing, A.S. Grader, P.M. Halleck, J. Am. Ceram. Soc. 89 (2006) 3027-3033.

[31] E.A. Olevsky, R.M. German, Acta Mater. 48 (2000) 1153-1166.

[32] E.A. Olevsky, R.M. German, A. Upadhyaya, Acta Mater. 48 (2000) 1167-1180.

[33] A. Wonisch, O. Guillon, T. Kraft, M. Moseler, H. Riedel, J. Rödel, Acta Mater. 55 (2007) 51875199.

[34] C.L. Martin, H. Camacho-Montes, L. Olmos, D. Bouvard, R.K. Bordia, J. Am. Ceram. Soc. 92 (2009) 1435-1441.

[35] T. Rasp, C. Jamin, A. Wonisch, T. Kraft, O. Guillon, J. Am. Ceram. Soc. 95 (2012) 586-592.

[36] S.-Y. Tzeng, J.-H. Jean, J. Am. Ceram. Soc. 85 (2002) 335-340.

[37] T.T. Molla, D.W. Ni, R. Bulatova, R. Bjørk, C. Bahl, N. Pryds, H.L. Frandsen, J. Am. Ceram. Soc. 97 (2014) 2965-2972.

[38] T.T. Molla, R. Bjørk, E. Olevsky, N. Pryds, H.L. Frandsen, Comput. Mater. Sci. 88 (2014) $28-36$.

[39] D.B. Marshall, J.J. Ratto, F.F. Lange, J. Am. Ceram. Soc. 74 (1991) 2979-2987.

[40] T. Chartier, T. Rouxel, J. Eur. Ceram. Soc. 17 (1997) 299-308.

[41] K.P. Plucknett, C.H. Cáceres, C. Hughes, D.S. Wilkinson, J. Am. Ceram. Soc. 77 (1994) 21452153.

[42] G. Magnani, A. Brillante, J. Eur. Ceram. Soc. 25 (2005) 3383-3392.

[43] D.J. Green, P.Z. Cai, G.L. Messing, J. Eur. Ceram. Soc. 19 (1999) 2511-2517.

[44] M. Menon, I.-W. Chen, J. Am. Ceram. Soc. 82 (1999) 3413-3421.

[45] M. Menon, I.-W. Chen, J. Am. Ceram. Soc. 82 (1999) 3422-3429.

[46] M. Menon, I.-W. Chen, J. Am. Ceram. Soc. 82 (1999) 3430 - 3440.

[47] I.-W. Chen, E.J. Winn, M. Menon, Mater. Sci. Eng. A 317 (2001) 226-235. 
[48] A. Dakskobler, T. Kosmač, J. Eur. Ceram. Soc. 24 (2004) 3351-3357.

[49] F. Cordisco, P.D. Zavattieri, L.G. Hector Jr., A.F. Bower, Eng. Fract. Mech. 131 (2014) 38-57.

[50] E.A. Olevsky, Mater. Sci. Eng. R Rep. 23 (1998) 41-100.

[51] G.W. Scherer, J. Am. Ceram. Soc. 67 (1984) 709-715.

[52] A. Kubota, M. Ashizuka, H. Hokazono, J. Ceram. Soc. Jpn. 102 (1994) 175-179.

[53] N. Soga, O.L. Anderson, J. Am. Ceram. Soc. 49 (1966) 355-359.

[54] M.F. Ashby, HIP 6.0 Backgroung Reading, University of Cambridge, Cambridge, 1990.

[55] D. Giuntini, E.A. Olevsky, C. Garcia-Cardona, A.L. Maximenko, M.S. Yurlova, C.D. Haines, D.G. Martin, D. Kapoor, Materials 6 (2013) 2612-2632.

[56] D. Giuntini, J. Raethel, M. Herrmann, A. Michaelis, E.A. Olevsky, J. Am. Ceram. Soc. 98 (2015) $3529-3537$.

[57] D. Giuntini, J. Raethel, M. Herrmann, A. Michaelis, E.A. Olevsky, J. Ceram. Soc. Jpn. 24 (2016), 403-414. 

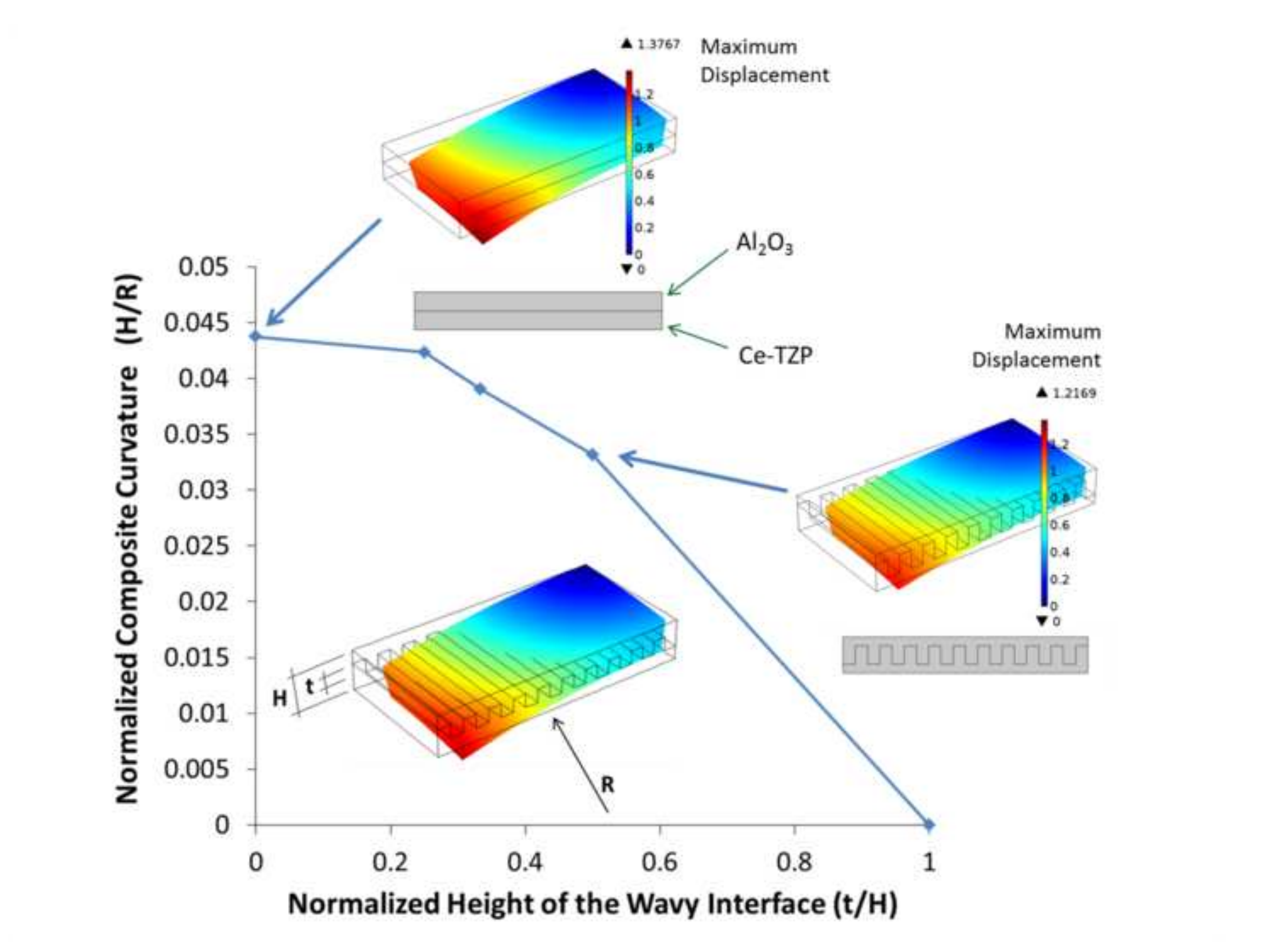

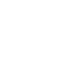

(

-

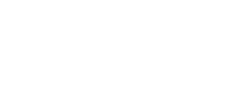

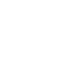

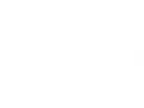

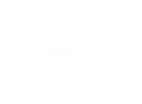

